\title{
Study on Chinese Semantic Content Based on Syntactic Differences Between Chinese and English
}

\author{
Feng $\mathrm{Li}^{1, *}$ \\ ${ }^{1}$ School of Foreign Languages, Jinling Institute of Technology, Nanjing, Jiangsu 211169, China \\ *Corresponding author.Email: phoenix@jit.edu.cn
}

\begin{abstract}
Many disputes appear between minimalists and contextualists in explaining semantic content. This article aims to explore Chinese semantic content based on syntactic differences between Chinese and English. The differences roughly include no tense for predicate verbs, no plural forms for nouns, no case change in forms for pronouns, no formal change between verbs and nouns in sentences, no subjects for many sentences, etc. Through analyses, it is revealed that though Chinese is very context-sensitive, both minimalism and contextualism account for its semantic content. These two linguistic views should not be split completely. Keywords: Semantic Content; Syntactic Differences; Minimalism; Contextualism
\end{abstract}

\section{INTRODUCTION}

Semantics, a study of meaning in language, is primarily linguistic, but also philosophical. It focuses on linguistic symbols, syntactically logical forms and contextual relations. This issue has been explored from various approaches, "from a pretty standard formal perspective at one end through to a pretty standard use-based perspective at the other"[1]. Fodor \& Lepore [2] believe that "the compositionality of natural languages explains how $\mathrm{L}$ (anguage)-speakers can understand any of the indefinitely many expressions that belongs to L", and they also claim that "compositionality excludes all 'pragmatist' accounts of content". So, minimalists [3] hold that semantic content for a sentence is fully determined by its syntactic structure and lexical content, i.e. the meaning of a sentence is exhausted by the meaning of its parts and their mode of composition. These theorists do not object to context effect completely, but they argue that context-sensitive expressions in natural language are very limited in number and usage.

On the contrary, according to contextualists [4], either moderately or radically, linguistic expressions carry different values in different contexts, and without a contribution from context they are intuitively felt to be 'incomplete'. In simple words, the context-independent content is non-existent. Contextualism cares about the speaker's intention and the listener's interpretation. In this sense, grasp of semantic content involves both 'word reading' and 'mind reading'. No matter how divergent they are, these approaches, from minimalism to contextualism, can avoid discussing neither lexico-syntactic content nor pragmatic content of a linguistic expression. As is known, most of the research has been conducted via European languages, mainly via English. Chinese language falls into a totally different family. How about Chinese when we study the meaning in Chinese?
Chinese is a different language from Indo-European language (narrowing down to English in this article). First, its writing system, unlike English, is ideographic, which means that usually Chinese characters composed of different strokes vary from each other in form. Such a writing system is much difficult to set down on paper compared with phonographic languages. Second, its pronunciation is unique from English. Most Chinese characters are one-syllable, which sounds easy to produce or comprehend. But these one-syllable pronunciations may be labeled by four different tones: level, rising, fallingrising, and falling. When a one-syllable pronunciation is labeled by the four tones, four characters with entirely different semantic contents are corresponded to each of the four sounds, though the four sounds sound quite similar only with a slight difference in tones. Such a pronunciation is hard to distinguish among the four tones even for native speakers.

The craziest part of Chinese, when compared with English, may lie in its grammar --- syntactic structure. For example, Chinese nouns do not have plural forms. So, when you see a noun in the sentence, you do not know there is only one 'this object' or there are many 'this object's from the lexico-syntactic elements. Chinese verbs do not possess inflectional changes (say, past tense, present tense, future tense, present participle, past participle, third person singular, etc.). Without extra information about time, you do not know whether an event happened, happens or will happen. In Chinese, nouns and verbs look the same in form. Sometimes we cannot judge whether an expression is a $\mathrm{VP}(\mathrm{V}+\mathrm{N})$ or a $\mathrm{NP}(\mathrm{N}+\mathrm{N})$ without contexts. For example, 阅 读 (n. or v.) 材料 (n.) can be interpreted as Comprehension materials OR Read the materials. Chinese adjectives do not own comparative degree or superlative degree in form, which requires additional auxiliary words to express comparative statements. And Chinese pronouns remain unchanged in form no matter whether acting as objective case, subjective case or possessive case. 
Of course, it may not be very reasonable to analyze Chinese language by means of English syntact. However, it is very significant and unprecedented to explore the issue of semantic content of Chinese language from the perspective of syntactic differences between Chinese and English, which, as the interest of this article, sheds a light on a new approach of research on minimalism and contextualism.

\section{DISTINCTIVE SYNTACTIC DIFFERENCES OF CHINESE COMPARED WITH ENGLISH}

Human perception and understanding of our living environment, namely human culture, exerts a great influence on what terminology we like to use, what implications we prefer, and what syntactic structure we employ to communicate [5]. Naturally, there exist both similarities and differences in how we perceive and understand this world across cultures. Therefore, it is understandable that languages always have something different as well as something in common even in syntactic level. Some distinctive variations arise in syntactics between Chinese and English, and this article will explore them and analyze the semantic content to see whether minimalists or contextualists apply to Chinese language.

\subsection{No Tense for Predicate Verbs in Chinese}

Chinese verbs do not undergo inflectional changes, such as present participle, past participle, third person singular, etc. Particularly, there is no tense change for predicate verbs in Chinese. A predicate verb alone cannot display past tense, present tense or future tense. Without additional information about time (or we can say without a certain context), we are unable to distinct whether an event took place, takes place or will take place.

e.g.

他 养 很多 猫。

He keep many cats

This Chinese sentence tells us no more than a proposition or a fact --- his keeping many cats. But in English, it can be translated as following:

He kept many cats.

He keeps many cats.

He will keep many cats.

Compared with the English sentences, the Chinese sentence is felt to be incomplete in meaning. The semantic content of each of the three English sentences can be better determined by its syntactic structure and lexical content while the semantic content of the Chinese sentence seems more context-sensitive.

\subsection{No Plural Forms for Nouns in Chinese}

In Chinese a noun remains the same formally either for singular meaning or for plural meaning.

e.g.

桌上有书。

In English this sentence may be interpreted as:

There is a book on the desk.

There are books on the desk.

'书' in Chinese does not change in form whether for ' a book' or 'many books'. There is no plural form 's , behind the Chinese character. So, syntactically, a noun in Chinese sentences need more information to achieve more complete meaning. Comparative speaking, English is much more context-independent than Chinese as far as nouns are concerned.

Sometimes, when there exist two nouns in a Chinese sentence, it is hard to decide which one their previous quantifier intends to modify.

e.g.

他买了两个孩子的玩具。

The quantifier '两个' may modify '孩子' as well as “玩具' . In the Chinese sentence we cannot determine whether 'there are two kids' or 'there are two toys'. In this case, ambiguity may arise, for more interpretation of this sentence can be produced pragmatically.

\subsection{No Case Change in Form for Pronouns in Chinese}

A Chinese pronoun does not distinguish subjective case from objective case formally. But this does not bother us to decide whether it is a subject or object, for the position of one pronoun can reveal its case.

e.g.

我爱人人，人人爱我。

I love everyone, and everyone loves me.

Put in the front of the sentence, '我' acts as a subject, while behind a transitive verb, it is an object. Syntactically, both the Chinese sentence can display its semantic content as much as the English sentence. But in some rare cases, humor emerges from ambiguity due to no change in form for interrogative pronouns such as '谁' .

e.g.

我单身的原因有两个:

There are two reasons why I am still single:

一是：谁都看不上;

One is whoever does not take a fancy to (me);

二是：谁都看不上。

The other is whomever (I) do not take fancy to. 
When ambiguity arises, Chinese sentences inspire more pragmatic content while English sentences rely on more lexico-syntactic content.

\subsection{No Formal Change between Verbs and Nouns in Chinese Sentences}

Chinese verbs can also be used as nouns. It is easy to confuse a Chinese verb with a noun if isolated from a certain context.

e.g.

这个门没有锁。

'锁' in the sentence can be understood as a noun (a lock) or a verb (be locked). In English there are also many words acting as nouns as well as verbs, such as 'lock' , 'smile', 'hug', etc. But their parts of speech can be clearly distinguished from a verb to a noun, for these words will display different grammatical forms in English sentences.

e.g.

The door does not have a lock. Or

The door is not locked.

This feature of no formal change between verbs and nouns in Chinese sentences renders Chinese more contextdependent.

\subsection{No Subjects for Many Sentences in Chinese}

In Chinese there are a lot of ellipsis in the sentences, especially the ellipsis of subjects, which is common in Chinese idioms, proverbs and poems.

e.g.

好好学习, 天天向上。

If put in English, it should be added with a proper subject. Work hard, and we will make progress every day.

A poem is illustrated as follow:

静夜思（李白）

床前明月光, 疑是地上霜。

举头望明月，低头思故乡!

A Tranquil Night (by Li Bai)

Before my bed a pool of light,

Is it hoarfrost upon the ground?

Eyes raised, I see the moon so bright;

Head bent, in homesick I'm drowned. --- taken from 300 Tang Poems

A New Translation

Despite no subject in the Chinses expressions, there is no confusion or difficulty for Chinese people to understand their semantic contents. The reasons may lie in two aspects: such expressions are articulated to all people in our culture instead of a specific group; and Chinese people are more context-sensitive in perceiving and understanding the world. Nisbett [6] found that the Chinese have developed a type of dialectical thought that seeks to use contradiction to understand relations and to see things in their appropriate contexts, which means that events do not occur in isolation from other events, but are instead embedded in a meaningful whole, while a Western dialectic is very "aggressive" in seeking to decontextualize and resolve contradictions. Therefore, the incomplete lexico-syntactic Chinese expressions contribute to many semantic contents, which is favored in Chinese culture.

\section{CONCLUSION}

Compared with English, there are such distinctive syntactic differences in Chinese as no tense for predicate verbs, no plural forms for nouns, no case change in forms for pronouns, no formal change between verbs and nouns in sentences, no subjects for many sentences, etc. These differences, on one hand, seem to make it impossible to grasp so-called true semantic content from a minimalist's view; one the other hand, they also seem to bring about a great mental burden in order to understand the semantic content from a contextualist's view, for too much contextual or background information is needed.

According to some radical contexualists, the English sentence "John weighs 80 pounds", which seems to be a very clearly semantic structure, can be interpreted differently in such contexts as 'with clothes', 'naked', 'on the earth' or 'on the moon'. So, those radical contextualists must get crazy when they try to deal with the meaning of crazy Chinese. But, obviously, Chinese, as the most ancient language still in use today, has been understood quite well in communication and transmission. So, what facilitate the meaning of Chinese sentences to be grasped? Chinese culture accepts contradictions and thinks in dialectic way. So Chinese thought is featured with context or situation specific, holistic, intuitive, and compatible. Western thought is fundamentally consistent with the laws of formal logic, usually characterized by being rule- or law-orientated, detailed, analytical and logical. Western thinking style determines that the grammar of English may be analytical, logical and less dependent on context, which requires it to possess some grammatical forms so that messages can be understood correctly even isolated from contexts. We may not conclude what exactly Chinese syntactic structure is supposed to be, but the Chinese thinking style tells us that its structure probably imposes less emphasis on grammatical forms, and more on language contexts and on wholeness of objects and events. Undoubtedly, interpretability and understandability of Chinese language are based on its compositionality, i.e. its lexico-syntactic content, which provides a general and genuine proposition to listeners or readers. But apparently, Chinese semantic content cannot be fully determined by its syntactic structure and lexical content. Grasp of Chinese semantic content involves both 'word reading' and 'mind reading', and the latter is much more context-sensitive. As 
Chinese thinking mode accepts contradictions, we accept both minimalism and contextualism to account for Chinese language. These two linguistic views should not be split completely. Instead, they should work together to explain natural languages in the world.

\section{REFERENCES}

[1] Borg, E. Pursuing Meaning. Oxford: Oxford University Press, 2012.

[2] Fodor, J. \& Lepore, E. The Compositionality Papers. Oxford: Oxford University Press, 2002.

[3] Borg, E. Minimalism versus Contextualism in Semantics. In G. Preyer, et al, (Eds.), Context- sensitivity and Semantic Minimalism Oxford: Oxford University Press, 2007, pp. 340-343.

[4] Recanati, F.. Literal Meaning. Journal of Linguistics, 2005, 41(3): 667-671.

[5] Li, F. Exploring Ambiguity Based on the Grammatical Differences between Chinese and English. Journal of Educational System, 2018, 2(1): 27-33.

[6] Nisbett, R. E. The Geography of Thought: How Asians and Westerners Think Differently and Why. New York: Free Press, 2003. 